\title{
Distinct Transport Regimes for Two Elastically Coupled Molecular Motors
}

\author{
Florian Berger, Corina Keller, Stefan Klumpp, and Reinhard Lipowsky \\ Theory and Bio-Systems, Max Planck Institute of Colloids and Interfaces, 14424 Potsdam, Germany
}

(Received 23 September 2011; published 14 May 2012)

\begin{abstract}
Cooperative cargo transport by two molecular motors involves an elastic motor-motor coupling, which can reduce the motors' velocity and/or enhance their unbinding from the filament. We show theoretically that these interference effects lead, in general, to four distinct transport regimes. In addition to a weak coupling regime, kinesin and dynein motors are found to exhibit a strong coupling and an enhanced unbinding regime, whereas myosin motors are predicted to attain a reduced velocity regime. All of these regimes, which we derive by explicit calculations and general time scale arguments, can be explored experimentally by varying the elastic coupling strength.
\end{abstract}

DOI: 10.1103/PhysRevLett.108.208101

PACS numbers: 87.16.Nn, 87.15.hj, 87.16.A-

Introduction.-The transport of intracellular cargo such as macromolecules, filaments, vesicles, and organelles relies on the cooperative action of small numbers of molecular motors [1,2]. Much effort has been devoted to study the transport behavior of a single motor molecule [3-5] and of large groups of motors working collectively [6-8], but transport by small teams of motors, as typically found in cells [1], has been addressed only more recently [9]. Such motors are, in general, elastically coupled via their cargo which could induce strain and interference effects such as a reduced velocity and/or binding time of the motors. However, recent studies have come to different conclusions about the relevance of such effects.

The transport of beads by several kinesin motors as experimentally observed in Ref. [10] was consistent with weak motor-motor interference, but more recent experiments with two kinesin motors coupled via a DNA scaffold $[11,12]$ provided evidence for strong motor-motor interference. Likewise, theoretical studies of the load-free case also appeared to be inconsistent, since appreciable interference effects were found in Refs. [13-16] but not in Refs. [9,17-19].

One fundamental aspect of cooperative transport by several molecular motors is the force balance between the motors, as required by Newton's third law. This force balance is, however, time-dependent and changes whenever one of the motors performs a mechanical step, thereby generating elastic strain forces between the motors; see Fig. 1. Here, we develop a systematic theory for this strain-induced force generation and its effects on the motor dynamics. We show that the system in Fig. 1 exhibits four different transport regimes: a weak coupling regime without motor-motor interference and three distinct interference

Published by the American Physical Society under the terms of the Creative Commons Attribution 3.0 License. Further distribution of this work must maintain attribution to the author(s) and the published article's title, journal citation, and DOI. regimes. Which regime is explored depends on the interplay between the single motor properties and the effective strength $K$ of the elastic coupling between the motors. Previous studies [9,13-19] differed in these parameters, which explains why the results of these studies appeared to be inconsistent.

Our theory starts from the experimentally determined single motor properties such as the stall force $F_{s}$, at which the single motor velocity vanishes, the detachment force $F_{d}$, at which the motor becomes likely to unbind from the filament, and the elastic coupling strength of a single motor stalk. We consider two motors attached to the cargo via their stalks (see Fig. 1), express the parameters of the combined system in terms of the single motor properties, and study the stochastic dynamics of this system.

Detailed calculations and time scale arguments show that the different transport regimes arise from the competition of three processes: (i) spontaneous motor unbinding in the absence of appreciable strain forces, (ii) strain-induced unbinding arising from the elastic interactions between the motors, and (iii) strain-induced stalling of the motors, which leads to a reduction of the cargo velocity. Which


FIG. 1 (color online). Two molecular motors coupled to a cargo via their elastic stalks or linkers. The motors step forward stochastically and stretch their linkers, thereby inducing an elastic interaction that generates the strain force $F$ [33]. Strong elastic coupling leads to a fast buildup of large forces, whereas weakly coupled motors experience only relatively small forces. 
of the two strain-induced processes is more likely to occur depends on the relative size of the detachment force $F_{d}$ and the stall force $F_{s}$. Thus, if $F_{d}$ is small compared to $F_{s}$, the buildup of strain forces will typically lead to strain-induced unbinding, and the system will then enter an enhanced unbinding regime for sufficiently large values of the effective elastic coupling strength $K$. On the other hand, if $F_{d}$ is large compared to $F_{s}$, strain-induced stalling is more likely to occur, and sufficiently large $K$ values lead to a reduced velocity regime. Finally, for $F_{d} \simeq F_{s}$ and large $K$, the system exhibits a strong coupling regime with both strain-induced unbinding and stalling.

Our Letter is organized as follows. After a brief review of single motor properties, we introduce our theoretical description of two elastically coupled motors, derive the different transport regimes, and calculate the characteristic time scales for strain-induced unbinding and stalling of the motors. By comparing these time scales with the time scale for spontaneous unbinding of a motor, we obtain an intuitive understanding of the different regimes. Finally, we apply our theory to specific motors such as kinesins, dyneins, and myosins. Our theory agrees with the available experimental data for kinesins [11] and predicts reduced velocity regimes for myosins.

Description of a single motor-We describe a single motor as a stochastic stepper with step size $l$, which steps forward with a force-dependent rate $\alpha(F)$ [20]. We relate this rate to the force-dependent velocity $\mathcal{V}(F)$ via $\alpha(F) \equiv$ $\mathcal{V}(F) / l$. Measured force-velocity relations differ between different motors and different studies [3-5]. However, in all cases the velocity decreases with increasing load until it vanishes at the stall force $F_{s}$. To capture this behavior, we focus on a piecewise linear form for the force-velocity relation: $\quad \mathcal{V}(F)=v\left[1-\left(F / F_{s}\right)\right]$ for $0<F<F_{s}$, $\mathcal{V}(F)=v$ under assisting forces $F \leq 0$ and $\mathcal{V}(F)=0$, for $F \geq F_{s}$. We also studied other functional forms of $\mathcal{V}(F)$, including other slopes for assisting forces; see [21]. The unbinding rate $\epsilon_{1}(F) \equiv \epsilon \exp \left(|F| / F_{d}\right)$ of a single motor increases exponentially with the absolute value of the force $F$ [3], which is rescaled by the detachment force $F_{d}$, where $\epsilon$ is the force free unbinding rate. Since we focus our study on 2-motor runs, during which both motors are active, all results are independent of the rebinding rate.

Motor-motor coupling.-We consider the two motor linkers as linear springs with spring constant or elastic coupling strength $\kappa$ as suggested by experimental data for kinesin-1 [22]. Nonlinear springs could be easily incorporated into our theory. Stochastic motor stepping leads to stretching or relaxation of the two linkers, which are connected in series and, thus, lead to the effective coupling strength $K=\kappa / 2$ [21,23]. If the linkers are stretched by a single motor step of size $l$, the motors experience the elastic strain force $F_{K} \equiv K l$. We choose a discrete state space where every state $(0) \ldots(N)$ is associated with the extension of the motor linkers. In every state $(i)$ the force
$F_{i} \equiv i F_{K}$ is exerted on one motor and the opposing force $-F_{i}$ on the other. Transitions between these states correspond to stretching and relaxing of the elastic linkers, which we relate to the motor stepping rates $\alpha$; see [21].

Because each motor can unbind from and rebind to the filament, the number of motors that actively pull the cargo varies with time [9]. Thus, we have to distinguish 1- and 2-motor runs, in which the cargo is actively pulled by one and two motors, respectively. Since the dynamics of 1 -motor runs is well characterized by single motor experiments [3-5], we will focus on 2-motor runs. Since each of the two motors may unbind from the filament, such a run is terminated with the rate $\omega_{\text {off }}(i) \equiv \epsilon_{1}\left(F_{i}\right)+\epsilon_{1}\left(-F_{i}\right)=$ $2 \epsilon \exp \left(\left|i F_{K}\right| / F_{d}\right)$. The unbound motor is taken to rebind with a relaxed linker.

Using these force-dependent parameters and the method in Ref. [24], we calculate the probabilities $P_{i}$ to find the system in state $i$ as well as the average velocity

$$
v_{2} \equiv \frac{1}{2} \sum_{i}\left[\mathcal{V}\left(i F_{K}\right)+\mathcal{V}\left(-i F_{K}\right)\right] P_{i}
$$

and the average binding time

$$
t_{2} \equiv\left(\sum_{i} \omega_{\text {off }}(i) P_{i}\right)^{-1}
$$

of the 2-motor runs; see [21]. For noninteracting motors, the binding time $t_{2}=t / 2$, i.e., half the binding time $t \equiv$ $1 / \epsilon$ of a single motor [9]. Moreover, once $v_{2}$ and $t_{2}$ are known, the properties of the overall cargo run can be determined by using the approach in Ref. [9]; see [21].

Different transport regimes.-The influence of elastic strain on stepping and unbinding is governed by the relative magnitude of the strain force $F_{K}$, the detachment force $F_{d}$, and the stall force $F_{s}$. It is then convenient to introduce the rescaled forces $f_{s} \equiv F_{s} / F_{K}$ and $f_{d} \equiv F_{d} / F_{K}$ and to present the results of our calculations as a function of $f_{s}$ and $f_{d}$; see Fig. 2, where we display contour plots for the average binding time $t_{2}$, as in Eq. (2), and the average velocity $v_{2}$, as in Eq. (1). For the remaining free parameters, we used the kinesin- 1 values $v / l \simeq 125 / \mathrm{s}$ and $\epsilon \simeq 1 / \mathrm{s}$ [3].

Inspection of Fig. 2 reveals four different transport regimes: (I) a weak coupling regime for large $f_{s}$ and large $f_{d}$ corresponding to $F_{K} \ll F_{d}$ and $F_{K} \ll F_{s}$, in which both the velocity $v_{2}$ and the binding time $t_{2}$ are hardly affected; (II) a strong coupling regime for small $f_{s}$ and small $f_{d}$ or $F_{K} \simeq F_{d} \simeq F_{s}$, which is characterized by a reduction of $v_{2}$ and $t_{2}$; (III) a reduced velocity regime for small $f_{s}$ and large $f_{d}$ or $F_{K} \simeq F_{s} \ll F_{d}$, in which $v_{2}$ is reduced without an effect on the binding time $t_{2}$ [25]; and (IV) an enhanced unbinding regime for $F_{K} \simeq F_{d} \ll F_{s}$ that is distinguished by a reduced binding time but an essentially unchanged velocity. The same regimes are also present for the transport properties of the overall cargo runs [21]. 

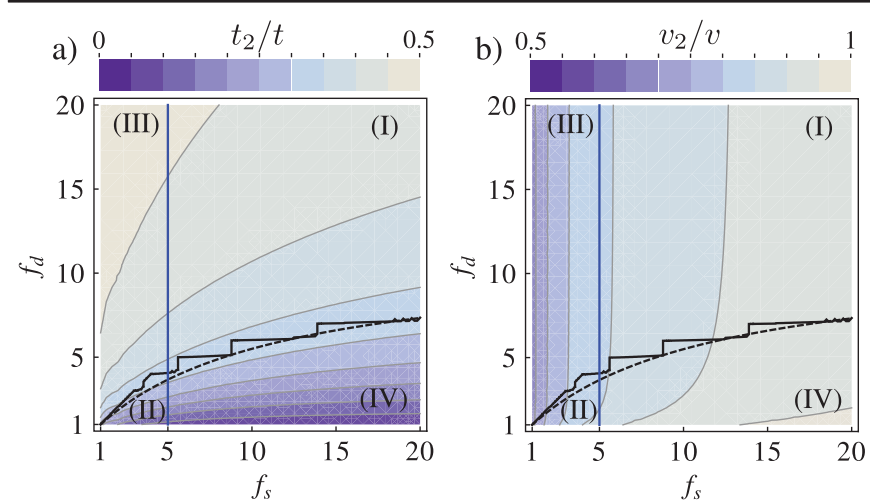

FIG. 2 (color). (a) Average binding time $t_{2}$ and (b) velocity $v_{2}$ of a cargo pulled by two active motors in units of the single motor binding time $t$ and single motor velocity $v$, respectively. Both quantities are displayed in a colored contour plot as functions of the rescaled single motor stall force $f_{s} \equiv F_{s} / F_{K}$ and the rescaled detachment force $f_{d} \equiv F_{d} / F_{K}$, where the force scale $F_{K}=K l$ is the elastic strain force between the motors per motor step. The solid blue line separates the regions with and without a reduced velocity, whereas the solid black line separates the regions of spontaneous and force-induced unbinding. These lines define four distinct transport regimes: (I) weak coupling with essentially unchanged velocity and binding time; (II) strong coupling with both reduced binding time and reduced velocity; (III) reduced velocity with no effect on the binding time; and (IV) enhanced unbinding with no effect on the velocity. The dashed lines correspond to the crossover line as given by (4).

Different time scales for strain force generation.-The dynamics of 2-motor runs, during which both motors collectively pull the cargo, is governed by the competition of several processes: spontaneous unbinding, strain-induced unbinding, and strain-induced stalling of a motor. In order to obtain some intuitive understanding of this competition, we now consider these processes separately and identify the characteristic time scales associated with them. Similar considerations have been used in the context of bond rupture $[26,27]$. Thus, we compare the following time scales: (i) the time $t_{u}$ for spontaneous unbinding of one of the two motors - this time is estimated by ignoring interactions between the motors and therefore given by $t_{u} \equiv 1 / 2 \epsilon$; (ii) the time $t_{F_{d}}$ required to build up elastic strain forces that are comparable to the detachment force; (iii) the time $t_{F_{s}}$ required to build up strain forces comparable to the stall force.

In order to estimate the time $t_{F_{d}}$, we neglect unbinding; i.e., we set all rates $\omega_{\text {off }}(i)=0$, consider two motors that start in the relaxed state (0), and calculate the mean first passage time for reaching the state $\left(\left[f_{d}\right]\right)$, in which the strain force is greater than or equal to the detachment force, where $\prod$ denotes the ceiling function. This time is given by

$$
t_{F_{d}}=\frac{l}{v} \sum_{i=0}^{\left\lceil f_{d}\right\rceil-1}\left(\frac{1}{2 \prod_{k=0}^{i}\left(1-\frac{k}{f_{s}}\right)}+\sum_{j=1}^{i} \frac{1}{\prod_{k=j}^{i}\left(1-\frac{k}{f_{s}}\right)}\right)
$$

for details, see [21]. The time $t_{F_{s}}$ is obtained from the same equation but with a different target state $\left(\left[f_{s}\right\rceil\right)$, in which the strain force is greater than or equal to the stall force.

The comparison of the two time scales $t_{u}$ and $t_{F_{d}}$ leads to two distinct unbinding regimes: (i) a regime of spontaneous unbinding for $t_{u}<t_{F_{d}}$, in which motors spontaneously unbind before the built-up of sufficiently large strain forces, and (ii) a regime of strain-induced unbinding for $t_{F_{d}}<t_{u}$. The crossover line between these two regimes is given by $t_{F_{d}}=t_{u}$; see the multiple-step functions in Figs. 2(a) and 2(b), which can be approximated [21] by

$$
f_{d}\left(f_{s}\right)=f_{d}^{*} f_{s} /\left(f_{d}^{*}+f_{s}-1\right) \text { with } f_{d}^{*} \equiv\lceil\sqrt{v / l \epsilon}\rceil,
$$

corresponding to the dashed black lines in Fig. 2.

From the comparison of the two time scales $t_{F_{s}}$ and $t_{u}$, we find two different velocity regimes: The velocity is substantially reduced for $t_{F_{s}}<t_{u}$ but hardly affected for $t_{F_{s}}>t_{u}$. The crossover line is obtained from $t_{F_{s}} \simeq t_{u}$, which leads to $f_{s}^{*} \sim\lceil\ln (v / 2 l \epsilon)\rceil[21]$; see the solid blue lines in Fig. 2. In terms of these time scales, the four transport regimes are now (I) weak coupling for $t_{u}<t_{F_{d}}$ and $t_{u}<t_{F_{s}}$; (II) strong coupling for $t_{u}>t_{F_{d}}$ and $t_{u}>t_{F_{s}}$; (III) reduced velocity for $t_{F_{s}}<t_{u}<t_{F_{d}}$; and (IV) enhanced unbinding for $t_{F_{d}}<t_{u}<t_{F_{s}}$. Note that, in addition to the force scales, the crossover lines also depend on the motor velocity $v$ and the unbinding rate $\epsilon$ of single motors. This dependence has its origin in the dynamic buildup of the mutual strain forces by single motor steps.

The values $f_{d}^{*}$ and $f_{s}^{*}$ decrease with decreasing $v / l \epsilon$, which is the single motor run length in units of the motor step size. Therefore, for highly processive motors with a large run length, such as kinesin-8 [28], the crossover lines are shifted to higher values of $f_{s}$ and $f_{d}$ leading to a reduced weak coupling regime. If the motors are slowed down, e.g., by decreasing the ATP concentration, the crossover lines are shifted to smaller values leading to an increased weak coupling region [21].

The time scales $t_{F_{d}}$ and $t_{F_{s}}$ also provide an intuitive understanding of the effects arising from the elastic coupling: For stiffer linkers, it takes less time to build up high strain forces between the motors, and thus the times $t_{F_{d}}$ and $t_{F_{s}}$ are rather small. In many models $[17,18]$ the motor linkers were described as cables to mimic a flexible polymer. With these cablelike linkers, a linear force-extension relation applies when the motor is extended beyond its rest length, but no force is built up if the linker is compressed, i.e., when the actual motor length is less than the rest length. When both motors start working together in a compressed state, no force is built up until their linkers are stretched. Therefore, in models with cablelike linkers, one typically has $t_{F_{d}}>t_{u}$ and $t_{F_{s}}>t_{u}$ and thus relatively small interference effects $[17,18]$.

Application to specific motors.-Finally, we use our framework to predict the behavior of pairs of different 
molecular motors. We fix the parameters describing these motors [21] and vary the elastic coupling strength $\kappa$, which leads to lines $f_{d}\left(f_{s}\right)=\left(F_{d} / F_{s}\right) f_{s}$ in the $\left(f_{s}, f_{d}\right)$ plane; see Fig. 3. These lines cross the different transport regimes, which are indicated by different colors. Pairs of kinesin-1 and weak dynein operate only in region (I), (II), or (IV), which have been previously discussed separately in the theoretical studies [9,13-18] for kinesin-1. Myosin V and myosin VI, on the other hand, are able to access only region (I) or (III), provided that $F_{d}>F_{s}$, as indicated by a recent estimate [29]. Thus, we predict that the main effect of coupling myosin motors is a reduced velocity.

In a recent study, two kinesin-1 motors have been connected via a rigid DNA scaffold with an elastic coupling strength of $\kappa \simeq 0.2 \mathrm{pN} / \mathrm{nm}$ [11]. This system is represented by the star on the kinesin- $1_{\mathrm{Di}}$ line in Fig. 3, which lies in the enhanced unbinding regime. Indeed, the motor pair studied in Ref. [11] was characterized by a strong reduction of the binding time, but essentially no effect on the cargo velocity as appropriate for this transport regime. After the submission of our Letter, we became aware of a recent study [30], in which two myosin V motors have been elastically coupled via a DNA scaffold and were observed to exhibit a reduced velocity, in agreement with our theory.

Summary.-We introduced a general theoretical framework to describe and understand cooperative cargo transport by molecular motors. The stochastic stepping of the motors leads to the buildup of elastic strain forces between the motors. However, the effects of these strain forces on the transport behavior depend on the magnitude of the effective elastic coupling $K$ as well as on the relative size of the stall force $F_{s}$ and the detachment force $F_{d}$, both of

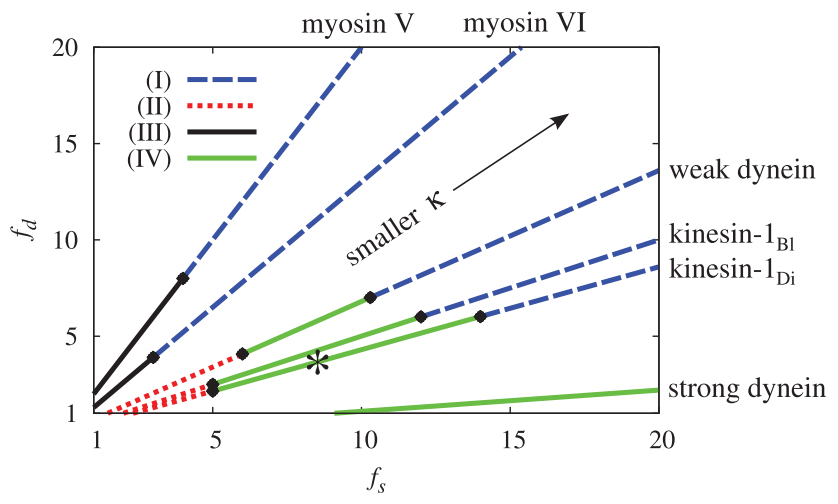

FIG. 3 (color). Transport regimes for pairs of different motors characterized by different straight lines $f_{d}\left(f_{s}\right)=\left(F_{d} / F_{s}\right) f_{s}$. We compare kinesin- $1_{\mathrm{B} 1}$ [3], kinesin-1 $1_{\mathrm{Di}}$ [11], weak dynein, strong dynein, myosin $\mathrm{V}$, and myosin $\mathrm{VI}$; the corresponding single motor parameters are described in Ref. [21]. The different transport regimes (I)-(IV) are color coded and separated by crossover points (black diamonds); the precise locations of these points are given in Ref. [21]. Each straight line can be explored by varying the elastic coupling strength $\kappa$ and, thus, $f_{s}=$ $F_{s} / l K=2 F_{s} / l \kappa$. The star indicates the pair of kinesin motors studied experimentally in Ref. [11]. which characterize the behavior of single motors. Indeed, our systematic theory for two elastically coupled motors reveals four different transport regimes: (I) weak coupling, (II) strong coupling, (III) reduced velocity, and (IV) enhanced unbinding; see Fig. 2. Using general time scale arguments, we also identify several competing processes that dominate in the different regimes: spontaneous motor unbinding in (I), strain-induced unbinding in (IV), straininduced stalling in (III), and both strain-induced unbinding and stalling in (II). Our theory is consistent with the available experimental data [11] and is able to predict the transport properties of pairs of identical motors from single motor parameters. As shown in Fig. 3, all transport regimes are accessible to experimental studies by varying the elastic coupling strength $\kappa$. Thus, future experiments using pairs of motors that are coupled by different elastic elements should be able to explore all of these regimes. Furthermore, the basic physical insight obtained here in terms of competing motor processes can now be used to elucidate cargo transport by larger motor teams as well as by two antagonistic teams of motors that perform a tug of war [31,32].

We thank Melanie J. I. Müller and Steffen Liepelt for stimulating discussions.

[1] S. P. Gross, M. Vershinin, and G. T. Shubeita, Curr. Biol. 17, R478 (2007).

[2] F. Berger, C. Keller, M. J. I. Müller, S. Klumpp, and R. Lipowsky, Biochem. Soc. Trans. 39, 1211 (2011).

[3] M. Schnitzer, K. Visscher, and S. Block, Nat. Cell Biol. 2, 718 (2000).

[4] S. Uemura, H. Higuchi, A. O. Olivares, E. M. De La Cruz, and S. Ishiwata, Nat. Struct. Mol. Biol. 11, 877 (2004).

[5] S. Toba, T. M. Watanabe, L. Yamaguchi-Okimoto, Y. Y. Toyoshima, and H. Higuchi, Proc. Natl. Acad. Sci. U.S.A. 103, 5741 (2006).

[6] S. Leibler and D. A. Huse, J. Cell Biol. 121, 1357 (1993).

[7] F. Jülicher and J. Prost, Phys. Rev. Lett. 75, 2618 (1995).

[8] P. Bieling, I. A. Telley, J. Piehler, and T. Surrey, EMBO Rep. 9, 1121 (2008).

[9] S. Klumpp and R. Lipowsky, Proc. Natl. Acad. Sci. U.S.A. 102, 17284 (2005).

[10] J. Beeg, S. Klumpp, R. Dimova, R. S. Gracià, E. Unger, and R. Lipowsky, Biophys. J. 94, 532 (2008).

[11] A. R. Rogers, J. W. Driver, P.E. Constantinou, D. K. Jamison, and M. R. Diehl, Phys. Chem. Chem. Phys. 11, 4882 (2009).

[12] D. K. Jamison, J. W. Driver, A. R. Rogers, P.E. Constantinou, and M.R. Diehl, Biophys. J. 99, 2967 (2010).

[13] Z. Wang and M. Li, Phys. Rev. E 80, 041923 (2009).

[14] C. Keller, Diploma thesis, Humboldt University of Berlin, 2009.

[15] J. W. Driver, A. R. Rogers, D. K. Jamison, R. K. Das, A. B. Kolomeisky, and M. R. Diehl, Phys. Chem. Chem. Phys. 12, 10398 (2010). 
[16] Y. Zhang, Phys. Rev. E 83, 011909 (2011).

[17] C. B. Korn, S. Klumpp, R. Lipowsky, and U. S. Schwarz, J. Chem. Phys. 131, 245107 (2009).

[18] A. Kunwar and A. Mogilner, Phys. Biol. 7, 016012 (2010).

[19] O. Campàs, Y. Kafri, K. B. Zeldovich, J. Casademunt, and J.-F. Joanny, Phys. Rev. Lett. 97, 038101 (2006).

[20] Backward steps can also be included but do not change our results substantially [21].

[21] See Supplemental Material at http://link.aps.org/ supplemental/10.1103/PhysRevLett.108.208101 for calculational details, additional plots, and chosen parameter values.

[22] K. Kawaguchi, S. Uemura, and S. Ishiwata, Biophys. J. 84, 1103 (2003).

[23] We implicitly assumed here that the cargo is rigid. For flexible or deformable cargos, the parameter $K$ includes contributions from the cargo's elasticity.

[24] T. L. Hill, Proc. Natl. Acad. Sci. U.S.A. 85, 2879 (1988).
[25] The reduced velocity regimes are absent for relations $\mathcal{V}(F)$ that are point-symmetric with respect to $F=0$; see [21].

[26] U. Seifert, Phys. Rev. Lett. 84, 2750 (2000).

[27] U.S. Schwarz, T. Erdmann, and I. B. Bischofs, BioSystems 83, 225 (2006).

[28] V. Varga, C. Leduc, V. Bormuth, S. Diez, and J. Howard, Cell 138, 1174 (2009).

[29] M. Y. Ali, G. G. Kennedy, D. Safer, K. M. Trybus, H. Lee Sweeney, and D. M. Warshaw, Proc. Natl. Acad. Sci. U.S.A. 108, E535 (2011).

[30] H. Lu, C. S. Bookwalter, E. B. Krementsova, M. R. Diehl, and K. M. Trybus, Biophys. J. 102, 568a (2012).

[31] M. J. I. Müller, S. Klumpp, and R. Lipowsky, Proc. Natl. Acad. Sci. U.S.A. 105, 4609 (2008).

[32] V. Soppina, A. K. Rai, A. J. Ramaiya, P. Barak, and R. Mallik, Proc. Natl. Acad. Sci. U.S.A. 106, 19381 (2009).

[33] The term "strain force" is used here as an abbreviation for "force induced by elastic strains." 